Mathematical Modelling and Analysis

Volume 19 Number 5, November 2014, 647-663

http://dx.doi.org/10.3846/13926292.2014.980345

(C) Vilnius Gediminas Technical University, 2014
Publisher: Taylor\&Francis and VGTU

http://www.tandfonline.com/TMMA

Print ISSN: 1392-6292

Online ISSN: 1648-3510

\title{
Boundary Value Problems for q-Difference Equations and Inclusions with Nonlocal and Integral Boundary Conditions
}

\author{
Bashir Ahmad ${ }^{a}$ and Sotiris K. Ntouyas ${ }^{b, *}$ \\ ${ }^{a}$ King Abdulaziz University, Department of Mathematics, Faculty of Science \\ P.O. Box 80203, 21589 Jeddah, Saudi Arabia \\ ${ }^{b}$ University of Ioannina, Department of Mathematics \\ 45110 Ioannina, Greece \\ E-mail: bashirahmad_qau@yahoo.com \\ E-mail(corresp.): sntouyas@uoi.gr
}

Received October 31, 2013; revised October 9, 2014; published online November 1, 2014

\begin{abstract}
In this paper, we study boundary value problems for $q$-difference equations and inclusions with nonlocal and integral boundary conditions. Some new existence and uniqueness results are obtained by using a variety of fixed point theorems. Examples are given to illustrate the results.
\end{abstract}

Keywords: $q$-difference equations, nonlocal conditions, integral boundary conditions, existence, fixed point.

AMS Subject Classification: 34A08; 26A33; 34A60.

\section{Introduction}

The study of $q$-difference equations, initiated by Jackson [22,23], Carmichael [11], Mason [27] and Adams [1] in the first quarter of 20th century, has been developed over the years, for instance, see $[15,17]$. The $q$-difference equations and operators have extensively been investigated in the context of quantum calculus. In fact, $q$-calculus (quantum calculus) has a rich history and the details of its basic notions, results and methods can be found in the text [24]. Apart from the traditional treatment of quantum calculus, many interesting questions and problems, especially from theoretical point of view, either remained open or were partially answered. In recent years, the topic has attracted the attention of several researchers and a variety of new results can be found in the papers $[2,3,4,5,6,8,13,14,16,18,21,26]$. However, there are many aspects of boundary value problems of $q$-difference equations that need attention. For

* Member of Nonlinear Analysis and Applied Mathematics (NAAM)-Research Group at King Abdulaziz University, Jeddah, Saudi Arabia. 
instance, $q$-difference equations with nonlocal and integral boundary conditions are yet to be addressed.

In this paper, motivated by some recent theoretical work on the topic, we study the existence and uniqueness of solutions continuous at 0 for a boundary value problem of nonlinear $q$-difference equations with nonlocal and integral boundary conditions given by

$$
\left\{\begin{array}{l}
D_{q}^{2} x(t)=f(t, x(t)), \quad t \in I_{q}, \\
x(0)=x_{0}+g(x), \quad x(1)=\alpha \int_{\mu}^{\nu} x(s) d_{q} s, \quad x_{0} \in \mathbb{R},
\end{array}\right.
$$

where $f \in C\left(I_{q} \times \mathbb{R}, \mathbb{R}\right)$ is such that $f(t, x(t))$ is continuous at $t=0, I_{q}=$ $\left\{q^{n}: n \in \mathbb{N}\right\} \cup\{0,1\}, q \in(0,1)$ is a fixed constant and $\mu, \nu \in I_{q}$ with $\mu<\nu$, that is, $\mu=q^{n_{1}}, \nu=q^{n_{2}}, n_{1}>n_{2}, n_{1}, n_{2} \in \mathbb{N}, g: C\left(I_{q}, \mathbb{R}\right) \rightarrow \mathbb{R}$ and $\alpha \neq \frac{1+q}{\nu^{2}-\mu^{2}}$. In (1.1), for the sake of brevity, we write $g(x)=g\left(x\left(t_{i}\right)\right), t_{i} \in I_{q}$, for example, $g(x)=\sum_{i=1}^{n} c_{i} x\left(t_{i}\right)$. We emphasize that the nonlocal conditions of the form $x(0)=x_{0}+g(x)$ are found to be more useful than the standard initial conditions to describe some physical phenomena. For more details we refer to the work by Byszewski $[9,10]$.

In Section 3, we present the existence results for the problem (1.1). The first result is based on Banach's contraction principle and the second one on a fixed point theorem due to O'Regan. Concrete examples are provided for the illustration of the established results.

In Section 4, we consider a multi-valued analogue of problem (1.1) given by

$$
\left\{\begin{array}{l}
D_{q}^{2} x(t) \in F(t, x(t)), \quad t \in I_{q} \\
x(0)=x_{0}+g(x), \quad x(1)=\alpha \int_{\mu}^{\nu} x(s) d_{q} s
\end{array}\right.
$$

where $F: I_{q} \times \mathbb{R} \rightarrow \mathcal{P}(\mathbb{R})$ is a multivalued map, $\mathcal{P}(\mathbb{R})$ is the family of all nonempty subsets of $\mathbb{R}$. The existence of solutions for the problem (1.2) is shown by applying the nonlinear alternative for contractive maps.

\section{Preliminaries}

Let us recall some basic concepts of $q$-calculus $[7,17,24]$.

Let $0<q<1$, and $f$ a function defined on a $q$-geometric set $A$, i.e. $q t \in A$ for all $t \in A$. The $q$-difference operator is defined by

$$
D_{q} f(t)= \begin{cases}\frac{f(t)-f(q t)}{(1-q) t}, & t \in A \backslash\{0\} \\ \lim _{n \rightarrow \infty} \frac{f\left(t q^{n}\right)-f(0)}{t q^{n}}, & t=0\end{cases}
$$

provided that the limit exists and does not depend on $t$. The higher order $q$-derivatives are given by

$$
D_{q}^{0} f(t)=f(t), \quad D_{q}^{n} f(t)=D_{q} D_{q}^{n-1} f(t), \quad n \in \mathbb{N} .
$$


The Jackson $q$-integration [22] is

$$
\int_{0}^{a} f(t) d_{q} t=a(1-q) \sum_{n=0}^{\infty} q^{n} f\left(a q^{n}\right), \int_{a}^{b} f(t) d_{q}(t)=\int_{0}^{b} f(t) d_{q}(t)-\int_{0}^{a} f(t) d_{q}(t)
$$

where $a, b \in A$, provided that the series converge. Here we remark that the integral $\int_{a}^{b} f(t) d_{q}(t)$ is understood as a right inverse of the $q$-derivative.

For $0 \in A, f$ is called $q$-regular at zero if $\lim _{n \rightarrow \infty} f\left(t q^{n}\right)=f(0)$ for every $t \in A, t \neq 0$. It is important to note that continuity at zero implies $q$-regularity at zero but the converse is not true (see an example on page 7 in [7]).

Definition 1. Let $f$ be a function defined on a $q$-geometric set $A$. Then $f$ is q-integrable on $A$ if and only if $\int_{0}^{t} f(\mu) d_{q} \mu$ exists for all $t \in A$.

The $q$-integration by parts rule is

$$
\int_{a}^{b} u(q t) D_{q} v(t) d_{q} t=u(b) v(b)-u(a) v(a)+\int_{a}^{b} D_{q} u(t) v(t) d_{q} t,
$$

provided that $u$ and $v$ are $q$-regular at zero functions.

Let $f$ be a $q$-regular at zero function defined on a $q$-geometric set $A$ containing zero. Then

$$
F(z)=\int_{c}^{z} f(s) d_{q} s, \quad z \in A
$$

is $q$-regular at zero, where $c$ is a fixed point in $A$. Furthermore, $D_{q} F(z)$ exists for every $z \in A$ and

$$
D_{q} F(z)=f(z), \quad z \in A .
$$

Conversely, if $a$ and $b$ are two points in $A$, then

$$
\int_{a}^{b} D_{q} f(s) d_{q} s=f(b)-f(a)
$$

In the sequel, we denote by $\mathcal{C}=C\left(I_{q}, \mathbb{R}\right)$ the space of all functions from $I_{q} \rightarrow \mathbb{R}$ which are continuous at 0 .

Lemma 1. Let $\alpha \neq \frac{1+q}{\nu^{2}-\mu^{2}}$ and let $y \in C\left(I_{q}, \mathbb{R}\right)$. Then boundary value problem

$$
\left\{\begin{array}{l}
D_{q}^{2} x(t)=y(t), \quad t \in I_{q}, \\
x(0)=x_{0}+g(x), \quad x(1)=\alpha \int_{\mu}^{\nu} x(s) d_{q} s, \quad \mu<\nu, \mu, \nu \in I_{q}
\end{array}\right.
$$

is equivalent to a q-integral equation

$$
\begin{aligned}
x(t)= & \int_{0}^{t}(t-q s) y(s) d_{q} s+\frac{\alpha(1+q) t}{1+q-\alpha\left(\nu^{2}-\mu^{2}\right)} \\
& \times \int_{\mu}^{\nu} \int_{0}^{s}(s-r q) y(r) d_{q} r d_{q} s-\frac{(1+q) t}{1+q-\alpha\left(\nu^{2}-\mu^{2}\right)} \int_{0}^{1}(1-q s) y(s) d_{q} s \\
& +\left[1+\frac{(1+q)(\alpha(\nu-\mu)-1) t}{1+q-\alpha\left(\nu^{2}-\mu^{2}\right)}\right]\left(x_{0}+g(x)\right)
\end{aligned}
$$


Proof. As argued in [7], a solution continuous at zero of the equation $D_{q}^{2} x(t)=$ $y(t)$ can be written as

$$
x(t)=\int_{0}^{t}(t-q s) y(s) d_{q} s+c_{0} t+c_{1}, \quad t \in I_{q},
$$

where $c_{0}, c_{1}$ are constants (for functions not necessarily continuous at zero, the constants $c_{0}, c_{1}$ are $q$-periodic functions [7]). Using the boundary conditions given in (2.1), we find that $c_{1}=x_{0}+g(x)$ and

$$
\begin{aligned}
c_{0}= & \frac{1+q}{1+q-\alpha\left(\nu^{2}-\mu^{2}\right)}\left[\alpha \int_{\mu}^{\nu} \int_{0}^{s}(s-r q) y(r) d_{q} r d_{q} s\right. \\
& \left.-\int_{0}^{1}(1-q s) y(s) d_{q} s+(\alpha(\nu-\mu)-1)\left(x_{0}+g(x)\right)\right] .
\end{aligned}
$$

Substituting $c_{0}, c_{1}$ in (2.3), we obtain (2.2). The converse follows by applying the operator $D_{q}$ on $(2.2)$ and using the $q$-integration by parts formula.

\section{Existence Results - The Single-Valued Case}

In view of Lemma 1 , we introduce an operator $\mathcal{Q}: \mathcal{C} \rightarrow \mathcal{C}$ as

$$
\begin{aligned}
(\mathcal{Q} x)(t)= & \int_{0}^{t}(t-q s) f(s, x(s)) d_{q} s \\
& +\frac{\alpha(1+q) t}{1+q-\alpha\left(\nu^{2}-\mu^{2}\right)} \int_{\mu}^{\nu} \int_{0}^{s}(s-r q) f(r, x(r)) d_{q} r d_{q} s \\
& -\frac{(1+q) t}{1+q-\alpha\left(\nu^{2}-\mu^{2}\right)} \int_{0}^{1}(1-q s) f(s, x(s)) d_{q} s \\
& +\left[1+\frac{(1+q)(\alpha(\nu-\mu)-1) t}{1+q-\alpha\left(\nu^{2}-\mu^{2}\right)}\right]\left(x_{0}+g(x)\right) .
\end{aligned}
$$

Observe that the operator $\mathcal{Q}$ defined by (3.1) is well defined in view of the definition of the function $f$ and (1.1) has a solution if the operator equation $\mathcal{Q} x=x$ has a fixed point.

Define two operators from $\mathcal{C} \rightarrow \mathcal{C}$ as

$$
\begin{aligned}
\left(\mathcal{Q}_{1} x\right)(t)= & \int_{0}^{t}(t-q s) f(s, x(s)) d_{q} s \\
& +\frac{\alpha(1+q) t}{1+q-\alpha\left(\nu^{2}-\mu^{2}\right)} \int_{\mu}^{\nu} \int_{0}^{s}(s-r q) f(r, x(r)) d_{q} r d_{q} s \\
& -\frac{(1+q) t}{1+q-\alpha\left(\nu^{2}-\mu^{2}\right)} \int_{0}^{1}(1-q s) f(s, x(s)) d_{q} s, \quad t \in I_{q}
\end{aligned}
$$

and

$$
\left(\mathcal{Q}_{2} x\right)(t)=\left[1+\frac{(1+q)(\alpha(\nu-\mu)-1) t}{1+q-\alpha\left(\nu^{2}-\mu^{2}\right)}\right]\left(x_{0}+g(x)\right), \quad t \in I_{q} .
$$


Clearly

$$
(\mathcal{Q} x)(t)=\left(\mathcal{Q}_{1} x\right)(t)+\left(\mathcal{Q}_{2} x\right)(t), \quad t \in I_{q} .
$$

In the forthcoming results, the functions $f$ and $g$ are taken to be the same as defined in the problem (1.1).

Theorem 1. Assume that

$\left(A_{1}\right)|f(t, x)-f(t, y)| \leq L|x-y|, \forall t \in I_{q}, L>0, x, y \in \mathbb{R}$

$\left(A_{2}\right) g: \mathcal{C} \rightarrow \mathbb{R}$ is such that $|g(u)-g(v)| \leq \ell\|u-v\| \forall u, v \in \mathcal{C}$ with

$$
\begin{gathered}
\ell<\left[1+\frac{(1+q)|\alpha(\nu-\mu)-1|}{\left|1+q-\alpha\left(\nu^{2}-\mu^{2}\right)\right|}\right]^{-1} ; \\
\left(A_{3}\right) \gamma=L\left\{\frac{1}{1+q}+\frac{|\alpha|}{\left|1+q-\alpha\left(\nu^{2}-\mu^{2}\right)\right|} \frac{\left(\nu^{3}-\mu^{3}\right)}{\left(1+q+q^{2}\right)}+\frac{1}{\left|1+q-\alpha\left(\nu^{2}-\mu^{2}\right)\right|}\right\} \\
+\ell\left[1+\frac{(1+q)|\alpha(\nu-\mu)-1|}{\left|1+q-\alpha\left(\nu^{2}-\mu^{2}\right)\right|}\right]<1 .
\end{gathered}
$$

Then the boundary value problem (1.1) has a unique solution.

Proof. In view of the definition of $\mathcal{Q}$ and assumptions $\left(A_{1}\right)$ and $\left(A_{2}\right)$, for $x, y \in \mathcal{C}$ and for each $t \in I_{q}$, we obtain

$$
\begin{aligned}
\mid(\mathcal{Q} x) & (t)-(\mathcal{Q} y)(t) \mid \\
\leq & \int_{0}^{t}(t-q s)|f(s, x(s))-f(s, y(s))| d_{q} s+\frac{|\alpha|(1+q)}{\left|1+q-\alpha\left(\nu^{2}-\mu^{2}\right)\right|} \\
& \times \int_{\mu}^{\nu}\left(\int_{0}^{s}(s-q m)|f(m, x(m))-f(m, y(m))| d_{q} m\right) d_{q} s \\
& +\frac{(1+q)}{\left|1+q-\alpha\left(\nu^{2}-\mu^{2}\right)\right|} \int_{0}^{1}(1-q s)|f(s, x(s))-f(s, y(s))| d_{q} s \\
+ & {\left[1+\frac{(1+q)|\alpha(\nu-\mu)-1|}{\left|1+q-\alpha\left(\nu^{2}-\mu^{2}\right)\right|}\right]|g(x)-g(y)| } \\
\leq & L\|x-y\|\left\{\int_{0}^{t}(t-q s) d_{q} s\right. \\
+ & \frac{|\alpha|(1+q)}{\left|1+q-\alpha\left(\nu^{2}-\mu^{2}\right)\right|} \int_{\mu}^{\nu}\left(\int_{0}^{s}(s-q m) d_{q} m\right) d_{q} s \\
+ & \left.\frac{(1+q)}{\left|1+q-\alpha\left(\nu^{2}-\mu^{2}\right)\right|} \int_{0}^{1}(1-q s) d_{q} s\right\} \\
+ & {\left[1+\frac{(1+q)|\alpha(\nu-\mu)-1|}{\left|1+q-\alpha\left(\nu^{2}-\mu^{2}\right)\right|}\right] \ell\|x-y\| } \\
\leq & L\left\{\frac{1}{1+q}+\frac{|\alpha|}{\left|1+q-\alpha\left(\nu^{2}-\mu^{2}\right)\right|} \frac{\left(\nu^{3}-\mu^{3}\right)}{\left(1+q+q^{2}\right)}\right. \\
+ & \left.\frac{1}{\left|1+q-\alpha\left(\nu^{2}-\mu^{2}\right)\right|}\right\}^{\mid x-y\left\|+\left[1+\frac{(1+q)|\alpha(\nu-\mu)-1|}{\left|1+q-\alpha\left(\nu^{2}-\mu^{2}\right)\right|}\right] \ell x-y\right\|,},
\end{aligned}
$$


and hence taking the norm on $I_{q}$ yields

$$
\|\mathcal{Q} x-\mathcal{Q} y\| \leq \gamma\|x-y\|
$$

As $\gamma<1$ by $\left(A_{3}\right)$, therefore $\mathcal{Q}$ is a contraction map from the space $\mathcal{C}$ into itself. Thus, the conclusion of the theorem follows by the contraction mapping principle (Banach fixed point theorem).

Next, we introduce a fixed point theorem due to O'Regan [28] which will be used to prove the next result.

Lemma 2. Denote by $U$ an open set in a closed, convex set $C$ of a Banach space $E$. Assume $0 \in U$. Also assume that $F(\bar{U})$ is bounded and that $F: \bar{U} \rightarrow C$ is given by $F=F_{1}+F_{2}$, in which $F_{1}: \bar{U} \rightarrow E$ is continuous and completely continuous and $F_{2}: \bar{U} \rightarrow E$ is a nonlinear contraction (i.e., there exists a nonnegative nondecreasing function $\phi:[0, \infty) \rightarrow[0, \infty)$ satisfying $\phi(z)<z$ for $z>0$, such that $\left\|F_{2}(x)-F_{2}(y)\right\| \leq \phi(\|x-y\|)$ for all $\left.x, y \in \bar{U}\right)$. Then, either

(C1) F has a fixed point $u \in \bar{U}$; or

(C2) there exist a point $u \in \partial U$ and $\lambda \in(0,1)$ with $u=\lambda F(u)$, where $\bar{U}$ and $\partial U$, respectively, represent the closure and boundary of $U$.

Let $\Omega_{r}=\{x \in \mathcal{C}:\|x\|<r\}$ and denote the maximum number by

$$
M_{r}=\max \left\{|f(t, x)|:(t, x) \in I_{q} \times[-r, r]\right\} .
$$

Theorem 2. Suppose that $\left(A_{1}\right)$ and $\left(A_{2}\right)$ hold. In addition we assume that

$\left(A_{4}\right) g(0)=0$

$\left(A_{5}\right)$ there exists a nonnegative function $p \in \mathcal{C}$ and a nondecreasing function $\psi:[0, \infty) \rightarrow[0, \infty)$ such that

$$
|f(t, u)| \leq p(t) \psi(|u|) \quad \text { for any }(t, u) \in I_{q} \times \mathbb{R}
$$

$\left(A_{6}\right) \sup _{r \in(0, \infty)} \frac{r}{k_{0}\left|x_{0}\right|+p_{0} \psi(r)}>\frac{1}{1-k_{0} \ell}$, where

$$
\begin{aligned}
p_{0}= & \int_{0}^{1}(1-q s) p(s) d_{q} s+\frac{|\alpha|(1+q)}{\left|1+q-\alpha\left(\nu^{2}-\mu^{2}\right)\right|} \int_{\mu}^{\nu} \int_{0}^{s}(s-r q) p(r) d_{q} r d_{q} s \\
& +\frac{(1+q)}{\left|1+q-\alpha\left(\nu^{2}-\mu^{2}\right)\right|} \int_{0}^{1}(1-q s) p(s) d_{q} s
\end{aligned}
$$

and

$$
k_{0}=1+\frac{(1+q)|\alpha(\nu-\mu)-1|}{\left|1+q-\alpha\left(\nu^{2}-\mu^{2}\right)\right|} .
$$

Then the boundary value problem (1.1) has at least one solution on $I_{q}$. 
Proof. Consider the operator $\mathcal{Q}: \mathcal{C} \rightarrow \mathcal{C}$ defined by (3.4), that is,

$$
(\mathcal{Q} x)(t)=\left(\mathcal{Q}_{1} x\right)(t)+\left(\mathcal{Q}_{2} x\right)(t), \quad t \in I_{q},
$$

where the operators $\mathcal{Q}_{1}$ and $\mathcal{Q}_{2}$ are respectively defined by (3.2) and (3.3).

From $\left(A_{6}\right)$ there exists a number $r_{0}>0$ such that

$$
\frac{r_{0}}{k_{0}\left|x_{0}\right|+p_{0} \psi\left(r_{0}\right)}>\frac{1}{1-k_{0} \ell} .
$$

We shall prove that the operators $\mathcal{Q}_{1}$ and $\mathcal{Q}_{2}$ satisfy all the conditions of Lemma 2. Our proof consists of a series of steps.

Step 1. The operator $\mathcal{Q}_{1}$ is continuous and completely continuous. We first show that $\mathcal{Q}_{1}\left(\bar{\Omega}_{r_{0}}\right)$ is bounded. For any $x \in \bar{\Omega}_{r_{0}}$, we have

$$
\begin{aligned}
\left\|\mathcal{Q}_{1} x\right\| \leq & \int_{0}^{t}(t-q s)|f(s, x(s))| d_{q} s \\
& +\frac{|\alpha|(1+q)}{\left|1+q-\alpha\left(\nu^{2}-\mu^{2}\right)\right|} \int_{\mu}^{\nu}\left(\int_{0}^{s}(s-q m)|f(m, x(m))| d_{q} m\right) d_{q} s \\
& +\frac{(1+q)}{\left|1+q-\alpha\left(\nu^{2}-\mu^{2}\right)\right|} \int_{0}^{1}(1-q s)|f(s, x(s))| d_{q} s \\
\leq & M_{r_{0}}\left\{\frac{1}{1+q}+\frac{|\alpha|}{\left|1+q-\alpha\left(\nu^{2}-\mu^{2}\right)\right|} \frac{\left(\nu^{3}-\mu^{3}\right)}{\left(1+q+q^{2}\right)}\right. \\
& \left.+\frac{1}{\left|1+q-\alpha\left(\nu^{2}-\mu^{2}\right)\right|}\right\} .
\end{aligned}
$$

This shows that $\mathcal{Q}_{1}\left(\bar{\Omega}_{r_{0}}\right)$ is uniformly bounded.

In addition, for any $t_{1}, t_{2} \in I_{q}$, we have

$$
\begin{aligned}
\left|\left(\mathcal{Q}_{1} x\right)\left(t_{2}\right)-\left(\mathcal{Q}_{1} x\right)\left(t_{1}\right)\right| \\
\leq\left|\int_{0}^{t_{2}}\left(t_{2}-q s\right) f(s, x(s)) d_{q} s-\int_{0}^{t_{1}}\left(t_{1}-q s\right) f(s, x(s)) d_{q} s\right| \\
\quad+\frac{|\alpha|(1+q)\left|t_{2}-t_{1}\right|}{1+q-\alpha\left(\nu^{2}-\mu^{2}\right)} \int_{\mu}^{\nu} \int_{0}^{s}(s-r q)|f(r, x(r))| d_{q} r d_{q} s \\
\quad+\frac{(1+q)\left|t_{2}-t_{1}\right|}{1+q-\alpha\left(\nu^{2}-\mu^{2}\right)} \int_{0}^{1}(1-q s)|f(s, x(s))| d_{q} s \\
\leq M_{r_{0}}\left|\int_{0}^{t_{1}}\left(t_{2}-t_{1}\right) d_{q} s\right|+M_{r_{0}}\left|\int_{t_{1}}^{t_{2}}\left(t_{2}-q s\right) d_{q} s\right| \\
\quad+M_{r_{0}} \frac{|\alpha|(1+q)\left|t_{2}-t_{1}\right|}{\left|1+q-\alpha\left(\nu^{2}-\mu^{2}\right)\right|} \int_{\mu}^{\nu} \int_{0}^{s}(s-r q) d_{q} r d_{q} s \\
\quad+M_{r_{0}} \frac{(1+q)\left|t_{2}-t_{1}\right|}{\left|1+q-\alpha\left(\nu^{2}-\mu^{2}\right)\right|} \int_{0}^{1}(1-q s) d_{q} s,
\end{aligned}
$$

which is independent of $x$, and tends to zero as $t_{2}-t_{1} \rightarrow 0$. Thus, $\mathcal{Q}_{1}$ is equicontinuous. Hence, Arzelá-Ascoli theorem applies and thereby we conclude 
that $\mathcal{Q}_{1}\left(\bar{\Omega}_{r_{0}}\right)$ is compact and hence completely continuous. Now, let $x_{n} \subset \bar{\Omega}_{r_{0}}$ with $\left\|x_{n}-x\right\| \rightarrow 0$. Then the limit $\left|x_{n}(t)-x(t)\right| \rightarrow 0$ is uniformly valid on $I_{q}$. From the uniform continuity of $f(t, x)$ on the compact set $I_{q} \times\left[-r_{0}, r_{0}\right]$, it follows that $\left\|f\left(t, x_{n}(t)\right)-f(t, x(t))\right\| \rightarrow 0$ uniformly on $I_{q}$. Hence $\| \mathcal{Q}_{1} x_{n}-$ $\mathcal{Q}_{1} x \| \rightarrow 0$ as $n \rightarrow \infty$ which proves the continuity of $\mathcal{Q}_{1}$. Hence Step 1 is complete.

Step 2. The operator $\mathcal{Q}_{2}: \bar{\Omega}_{r_{0}} \rightarrow \mathcal{C}$ is contractive. This is a consequence of $\left(A_{2}\right)$. Indeed, we have

$$
\begin{aligned}
\left|\left(\mathcal{Q}_{2} x\right)(t)-\left(\mathcal{Q}_{2} y\right)(t)\right| & =\left|\left[1+\frac{(1+q)(\alpha(\nu-\mu)-1) t}{1+q-\alpha\left(\nu^{2}-\mu^{2}\right)}\right][g(x)-g(y)]\right| \\
& \leq\left[1+\frac{(1+q)|\alpha(\nu-\mu)-1|}{\left|1+q-\alpha\left(\nu^{2}-\mu^{2}\right)\right|}\right] \ell\|x-y\| .
\end{aligned}
$$

Taking the supremum over $t \in I_{q}$, we get

$$
\left\|\mathcal{Q}_{2} x-\mathcal{Q}_{2} y\right\| \leq L_{0}\|x-y\|, \quad L_{0}:=\left[1+\frac{(1+q)|\alpha(\nu-\mu)-1|}{\left|1+q-\alpha\left(\nu^{2}-\mu^{2}\right)\right|}\right] \ell<1 .
$$

Step 3. The set $\mathcal{Q}\left(\bar{\Omega}_{r_{0}}\right)$ is bounded. By $\left(A_{2}\right)$ and $\left(A_{4}\right)$, we get

$$
\left\|\mathcal{Q}_{2}(x)\right\| \leq\left(1+\frac{(1+q)|\alpha(\nu-\mu)-1|}{\left|1+q-\alpha\left(\nu^{2}-\mu^{2}\right)\right|}\right)\left(\left|x_{0}\right|+\ell r_{0}\right)
$$

for any $x \in \bar{\Omega}_{r_{0}}$. This, with the boundedness of the set $\mathcal{Q}_{1}\left(\bar{\Omega}_{r_{0}}\right)$ implies that the set $\mathcal{Q}\left(\bar{\Omega}_{r_{0}}\right)$ is bounded.

Step 4. The case $(\mathrm{C} 2)$ in Lemma 2 does not hold. To this end, we suppose that (C2) holds. Then, we have that there exist $\lambda \in(0,1)$ and $x \in \partial \Omega_{r_{0}}$ such that $x=\lambda \mathcal{Q} x$. So, we have $\|x\|=r_{0}$ and

$$
\begin{aligned}
x(t)= & \lambda \int_{0}^{t}(t-q s) f(s, x(s)) d_{q} s \\
& +\lambda \frac{\alpha(1+q) t}{1+q-\alpha\left(\nu^{2}-\mu^{2}\right)} \int_{\mu}^{\nu} \int_{0}^{s}(s-r q) f(r, x(r)) d_{q} r d_{q} s \\
& -\lambda \frac{(1+q) t}{1+q-\alpha\left(\nu^{2}-\mu^{2}\right)} \int_{0}^{1}(1-q s) f(s, x(s)) d_{q} s \\
& +\lambda\left[1+\frac{(1+q)(\alpha(\nu-\mu)-1) t}{1+q-\alpha\left(\nu^{2}-\mu^{2}\right)^{2}}\right]\left(x_{0}+g(x)\right) .
\end{aligned}
$$

With hypotheses $\left(A_{4}\right)-\left(A_{6}\right)$, we have

$$
\begin{aligned}
r_{0} \leq & \psi\left(r_{0}\right)\left\{\int_{0}^{1}(1-s) p(s) d_{q} s\right. \\
& +\frac{|\alpha|(1+q)}{\left|1+q-\alpha\left(\nu^{2}-\mu^{2}\right)\right|} \int_{\mu}^{\nu} \int_{0}^{s}(s-r q) p(r) d_{q} r d_{q} s \\
& \left.+\frac{(1+q)}{\left|1+q-\alpha\left(\nu^{2}-\mu^{2}\right)\right|} \int_{0}^{1}(1-q s) p(s) d_{q} s\right\} \\
& +\left[1+\frac{(1+q)|\alpha(\nu-\mu)-1|}{\left|1+q-\alpha\left(\nu^{2}-\mu^{2}\right)\right|}\right]\left(\left|x_{0}\right|+\ell r_{0}\right),
\end{aligned}
$$


which implies that

$$
r_{0} \leq k_{0} \ell r_{0}+k_{0}\left|x_{0}\right|+p_{0} \psi\left(r_{0}\right)
$$

In consequence,

$$
\frac{r_{0}}{k_{0}\left|x_{0}\right|+p_{0} \psi\left(r_{0}\right)} \leq \frac{1}{1-k_{0} \ell},
$$

which contradicts (3.5). Thus, the operators $\mathcal{Q}_{1}$ and $\mathcal{Q}_{2}$ satisfy all the conditions of Lemma 2. Hence, the operator $\mathcal{Q}$ has at least one fixed point $x \in \bar{\Omega}_{r_{0}}$, which is the solution of the problem (1.1).

Now we give concrete examples for the illustration of our results.

Example 1. Consider the following boundary value problem

$$
\left\{\begin{array}{l}
D_{q}^{2} x(t)=\frac{1}{4} \frac{1}{(t+2)^{2}} \cdot \frac{|x|}{1+|x|}+1+\sin ^{2} t, \quad t \in I_{q}, \\
x(0)=1+\frac{1}{16} x\left(t_{i}\right), \quad x(1)=4 \int_{1 / 3}^{1 / 2} x(s) d s, \quad t_{i} \in I_{q} .
\end{array}\right.
$$

Here $\ell=1 / 16, \alpha=4, \mu=1 / 3, \nu=1 / 2$, and $f(t, x)=\frac{1}{4} \frac{1}{(t+2)^{2}} \cdot \frac{|x|}{1+|x|}+1+\sin ^{2} t$. As $|f(t, x)-f(t, y)| \leq \frac{1}{16}|x-y|$, therefore $\left(A_{1}\right)$ is satisfied with $L=\frac{1}{16}$. Using the given data together with the assumption $\left(A_{3}\right): \gamma<1$, we obtain

$$
\frac{2+q}{1+q}+\frac{19+18(4+q)\left(1+q+q^{2}\right)}{6(4+9 q)\left(1+q+q^{2}\right)}<16
$$

which is true for $0<q<1$. Thus, all the conditions of Theorem 1 are satisfied. Hence, the conclusion of Theorem 1 applies to the problem (3.6).

Example 2. Let $\beta>0$ and consider the following problem

$$
\left\{\begin{array}{l}
D_{q}^{2} x(t)=\beta \sin ^{2} x, \quad t \in I_{q} \\
x(0)=\frac{1}{4}+\ell x\left(t_{i}\right), \quad x(1)=4 \int_{1 / 3}^{1 / 2} x(s) d s, \quad t_{i} \in I_{q}
\end{array}\right.
$$

where $\beta=3 / 25, \ell=1 / 25, \mu=1 / 3, \nu=1 / 2$. We shall show that the problem (3.7) admits at least one solution. Clearly $|f(t, x)| \leq\left|\beta \sin ^{2} x\right| \leq \beta x^{2}$, for any $(t, x) \in I_{q} \times \mathbb{R}$. Setting $p(t)=\beta$ and $\psi(x)=x^{2}$, it is easy to find that

$$
p_{0}=\left[\frac{1}{1+q}+\frac{9}{4+9 q}\left(\frac{19}{54\left(1+q+q^{2}\right)}+1\right)\right] \beta, \quad k_{0}=\frac{7+12 q}{4+9 q}
$$

while the condition $\left(A_{6}\right)$ gives $\sqrt{k_{0} p_{0}}+k_{0} \ell<1$, which holds for $0<q<1$. Therefore, by Theorem 2, we conclude that problem (3.7) has at least one solution on $I_{q}$. 


\section{Existence Results - The Multi-Valued Case}

First of all, we describe basic notions of multi-valued maps and fix our terminology $[12,20]$.

For a normed space $(X,\|\cdot\|)$, we define $\mathcal{P}_{b}(X)=\{Y \in \mathcal{P}(X): Y$ is bounded $\}$ and

$$
\mathcal{P}_{c p, c}(X)=\{Y \in \mathcal{P}(X): Y \text { is compact and convex }\} .
$$

A multi-valued map $\mathcal{G}: X \rightarrow \mathcal{P}(X)$ is convex (closed) valued if $\mathcal{G}(x)$ is convex (closed) for all $x \in X$. The map $\mathcal{G}$ is bounded on bounded sets if $\mathcal{G}(\mathbb{B})=$ $\bigcup_{x \in \mathbb{B}} \mathcal{G}(x)$ is bounded in $X$ for all $\mathbb{B} \in \mathcal{P}_{b}(X)$ (i.e. $\sup _{x \in \mathbb{B}}\{\sup \{|y|: y \in$ $\mathcal{G}(x)\}\}<\infty)$. $\mathcal{G}$ is called upper semi-continuous (u.s.c.) on $X$ if for each $x_{0} \in X$, the set $\mathcal{G}\left(x_{0}\right)$ is a nonempty closed subset of $X$, and for each open set $N$ of $X$ containing $\mathcal{G}\left(x_{0}\right)$, there exists an open neighborhood $\mathcal{N}_{0}$ of $x_{0}$ such that $\mathcal{G}\left(\mathcal{N}_{0}\right) \subseteq N . \mathcal{G}$ is said to be completely continuous if $\mathcal{G}(\mathbb{B})$ is relatively compact for every $\mathbb{B} \in P_{b}(X)$. If the multi-valued map $\mathcal{G}$ is completely continuous with nonempty compact values, then $\mathcal{G}$ is u.s.c. if and only if $\mathcal{G}$ has a closed graph, i.e., $x_{n} \rightarrow x_{*}, y_{n} \rightarrow y_{*}, y_{n} \in \mathcal{G}\left(x_{n}\right)$ imply $y_{*} \in \mathcal{G}\left(x_{*}\right)$.

Let $L^{1}\left(I_{q}\right)$ denote the space of all functions $f$ defined on $I_{q}$ such that $\|x\|_{L^{1}}=\int_{0}^{1}|x(t)| d t<\infty$.

Definition 2. A multivalued map $F: I_{q} \times \mathbb{R} \rightarrow \mathcal{P}(\mathbb{R})$ is said to be Carathéodory (in the sense of $q$-calculus) if $x \longmapsto F(t, x)$ is upper semicontinuous on $I_{q}$. Further a Carathéodory function $F$ is called $L^{1}$-Carathéodory if there exists $\varphi_{\alpha} \in L^{1}\left(I_{q}, \mathbb{R}^{+}\right)$such that $\|F(t, x)\|=\sup \{|v|: v \in F(t, x)\} \leq \varphi_{\alpha}(t)$ for all $\|x\| \leq \alpha$ on $I_{q}$ for each $\alpha>0$.

Definition 3. A function $x \in \mathcal{C}$ is a solution of the problem (1.2) if $x(0)=$ $x_{0}+g(x), x(1)=\alpha \int_{\mu}^{\nu} x(s) d_{q} s$ and there exists a function $f \in L^{1}\left(I_{q}, \mathbb{R}\right)$ such that it is continuous at $t=0$ and $f(t) \in F(t, x(t))$ on $I_{q}$, and

$$
\begin{aligned}
x(t)= & \int_{0}^{t}(t-q s) f(s) d_{q} s+\frac{\alpha(1+q) t}{1+q-\alpha\left(\nu^{2}-\mu^{2}\right)} \int_{\mu}^{\nu} \int_{0}^{s}(s-r q) f(r) d_{q} r d_{q} s \\
& -\frac{(1+q) t}{1+q-\alpha\left(\nu^{2}-\mu^{2}\right)} \int_{0}^{1}(1-q s) f(s) d_{q} s \\
& +\left[1+\frac{(1+q)(\alpha(\nu-\mu)-1) t}{1+q-\alpha\left(\nu^{2}-\mu^{2}\right)}\right]\left(x_{0}+g(x)\right) .
\end{aligned}
$$

For each $y \in \mathcal{C}$, define the set of selections of $F$ by

$$
S_{F, y}:=\left\{v \in \mathcal{C}: v(t) \in F(t, y(t)) \text { on } I_{q}\right\} .
$$

The following lemma will be used in the sequel.

Lemma 3. [25] Let $X$ be a Banach space. Let $F: J \times X \rightarrow \mathcal{P}_{c p, c}(X)$ be an $L^{1}$-Carathéodory multivalued map and let $\Theta$ be a linear continuous mapping from $L^{1}(J, X)$ to $C(J, X)$. Then the operator

$$
\Theta \circ S_{F}: C(J, X) \rightarrow \mathcal{P}_{c p, c}(C(J, X)), \quad x \mapsto\left(\Theta \circ S_{F}\right)(x)=\Theta\left(S_{F, x}\right)
$$

is a closed graph operator in $C(J, X) \times C(J, X)$. 
To prove our main result in this section we will use the following form of the nonlinear alternative for contractive maps [29, Corollary 3.8].

Theorem 3. Let $X$ be a Banach space, and $D$ be a bounded neighborhood of $0 \in X$. Let $Z_{1}: X \rightarrow \mathcal{P}_{c p, c}(X)$ and $Z_{2}: \bar{D} \rightarrow \mathcal{P}_{c p, c}(X)$ be two multi-valued operators satisfying

(a) $Z_{1}$ is contraction, and

(b) $Z_{2}$ is u.s.c. and compact.

Then, if $G=Z_{1}+Z_{2}$, either

(i) $G$ has a fixed point in $\bar{D}$ or

(ii) there is a point $u \in \partial D$ and $\lambda \in(0,1)$ with $u \in \lambda G(u)$.

Theorem 4. Assume that

$\left(H_{1}\right) F: I_{q} \times \mathbb{R} \rightarrow \mathcal{P}_{c p, c}(\mathbb{R})$ is such that $x \rightarrow F(t, x)$ is u.s.c. on $I_{q}$;

$\left(H_{2}\right)$ there exists a continuous nondecreasing function $\psi:[0, \infty) \rightarrow(0, \infty)$ and a function $p \in C\left(I_{q}, \mathbb{R}^{+}\right)$such that

$\|F(t, x)\|_{\mathcal{P}}:=\sup \{|y|: y \in F(t, x)\} \leq p(t) \psi(|x|) \quad$ for each $(t, x) \in I_{q} \times \mathbb{R} ;$

$\left(H_{3}\right) g$ satisfies $\left(A_{2}\right)$;

$\left(H_{4}\right)$ there exists a number $M>0$ such that

$$
\frac{\left(1-\ell \Lambda_{1}\right) M}{\Lambda_{2} \psi(M)\|p\|+\Lambda_{1}\left|x_{0}\right|}>1, \ell \Lambda_{1}<1,
$$

where

$$
\begin{gathered}
\|p\|=\sup _{t \in I_{q}}|p(t)|, \quad \Lambda_{1}=1+\frac{(1+q)|\alpha(\nu-\mu)-1|}{\left|1+q-\alpha\left(\nu^{2}-\mu^{2}\right)\right|}, \\
\Lambda_{2}=\frac{1}{1+q}+\frac{|\alpha|}{\left|1+q-\alpha\left(\nu^{2}-\mu^{2}\right)\right|} \frac{\nu^{3}-\mu^{3}}{\left(1+q+q^{2}\right)}+\frac{1}{\left|1+q-\alpha\left(\nu^{2}-\mu^{2}\right)\right|} .
\end{gathered}
$$

Then the boundary value problem (1.2) has at least one solution on $I_{q}$.

Proof. To transform the problem (1.2) into a fixed point problem, we define an operator $\mathcal{N}: \mathcal{C} \longrightarrow \mathcal{P}(\mathcal{C})$ as

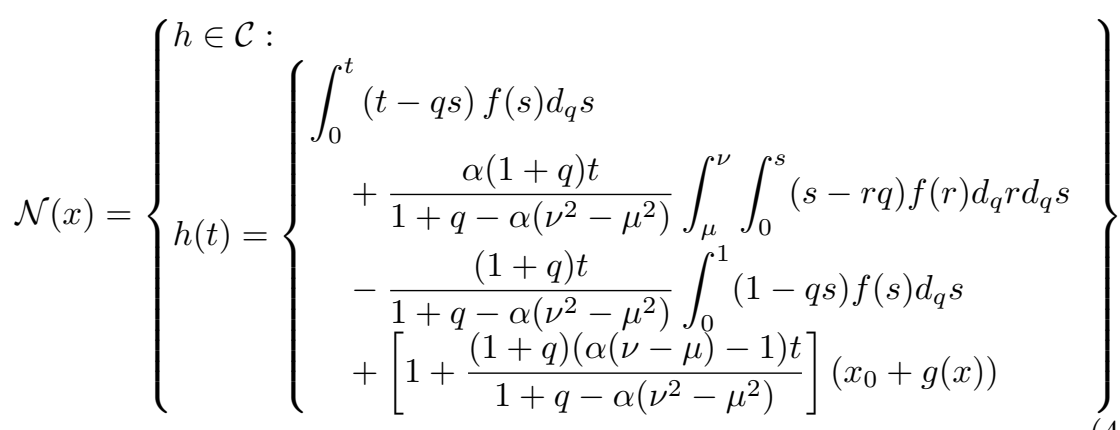


for $f \in S_{F, x}$.

Next, we introduce two operators $\mathcal{A}: \mathcal{C} \longrightarrow \mathcal{C}$ and $\mathcal{B}: \mathcal{C} \longrightarrow \mathcal{P}(\mathcal{C})$ as follow:

$$
\begin{gathered}
\mathcal{A} x(t)=\left[1+\frac{(1+q)(\alpha(\nu-\mu)-1) t}{1+q-\alpha\left(\nu^{2}-\mu^{2}\right)}\right]\left(x_{0}+g(x)\right), \\
h\left(x \in \mathcal{C}:\left\{\begin{array}{c}
\int_{0}^{t}(t-q s) f(s) d_{q} s \\
+\frac{\alpha(1+q) t}{1+q-\alpha\left(\nu^{2}-\mu^{2}\right)} \int_{\mu}^{\nu} \int_{0}^{s}(s-r q) f(r) d_{q} r d_{q} s \\
-\frac{(1+q) t}{1+q-\alpha\left(\nu^{2}-\mu^{2}\right)} \int_{0}^{1}(1-q s) f(s) d_{q} s
\end{array}\right\} .\right.
\end{gathered}
$$

Observe that $\mathcal{N}=\mathcal{A}+\mathcal{B}$. We shall show that the operators $\mathcal{A}$ and $\mathcal{B}$ satisfy all the conditions of Theorem 3 on $I_{q}$. For the sake of clarity, split the proof into a number of steps and claims.

Step 1. $\mathcal{A}$ is a contraction on $\mathcal{C}$. The proof is similar to the one for the operator $\mathcal{Q}_{2}$ in Step 2 of Theorem 2 .

Step 2. $\mathcal{B}$ is compact, convex valued and completely continuous. This will be established in several claims.

Claim I. $\mathcal{B}$ maps bounded sets into bounded sets in $\mathcal{C}$. For that, let $B_{r}=$ $\{x \in \mathcal{C}:\|x\| \leq r\}$ be a bounded set in $\mathcal{C}$. Then, for each $h \in \mathcal{B}(x), x \in B_{r}$, there exists $f \in S_{F, x}$ such that

$$
\begin{aligned}
|h(t)| \leq & \frac{1}{q+1}+\frac{|\alpha|(1+q)}{\left|1+q-\alpha\left(\nu^{2}-\mu^{2}\right)\right|} \int_{\mu}^{\nu} \int_{0}^{s}(s-r q)|f(r)| d_{q} r d_{q} s \\
& +\frac{(1+q)}{\left|1+q-\alpha\left(\nu^{2}-\mu^{2}\right)\right|} \int_{0}^{1}(1-q s)|f(s)| d_{q} s \\
\leq & \left\{\frac{1}{1+q}+\frac{|\alpha|}{\left|1+q-\alpha\left(\nu^{2}-\mu^{2}\right)\right|} \frac{\left(\nu^{3}-\mu^{3}\right)}{\left(1+q+q^{2}\right)}\right. \\
& \left.+\frac{1}{\left|1+q-\alpha\left(\nu^{2}-\mu^{2}\right)\right|}\right\} \psi(\|x\|)\|p\| .
\end{aligned}
$$

Consequently, for each $h \in \mathcal{B}\left(B_{q}\right)$, we have

$$
\begin{aligned}
\|h\| \leq & \left\{\frac{1}{1+q}+\frac{|\alpha|}{\left|1+q-\alpha\left(\nu^{2}-\mu^{2}\right)\right|} \frac{\left(\nu^{3}-\mu^{3}\right)}{\left(1+q+q^{2}\right)}\right. \\
& \left.+\frac{1}{\left|1+q-\alpha\left(\nu^{2}-\mu^{2}\right)\right|}\right\} \psi(r)\|p\| .
\end{aligned}
$$

Claim II. $\mathcal{B}$ maps bounded sets into equi-continuous sets. As before, let $B_{r}$ be a bounded set and let $h \in \mathcal{B}(x)$ for $x \in B_{r}$. Let $t_{1}, t_{2} \in I_{q}$ and $x \in B_{r}$. For 
each $h \in \mathcal{B}(x)$, we obtain

$$
\begin{aligned}
\left|h\left(t_{2}\right)-h\left(t_{1}\right)\right| \leq & \left|\int_{0}^{t_{2}}\left(t_{2}-q s\right) f(s) d_{q} s-\int_{0}^{t_{1}}\left(t_{1}-q s\right) f(s) d_{q} s\right| \\
& +\frac{|\alpha|(1+q)\left|t_{2}-t_{1}\right|}{\left|1+q-\alpha\left(\nu^{2}-\mu^{2}\right)\right|} \int_{\mu}^{\nu} \int_{0}^{s}(s-r q)|f(r)| d_{q} r d_{q} s \\
& +\frac{(1+q)\left|t_{2}-t_{1}\right|}{\left|1+q-\alpha\left(\nu^{2}-\mu^{2}\right)\right|} \int_{0}^{1}(1-q s)|f(s)| d_{q} s .
\end{aligned}
$$

Obviously the right hand side of the above inequality tends to zero independently of $x \in B_{r}$ as $t_{2}-t_{1} \rightarrow 0$. Therefore it follows by the Arzelá-Ascoli theorem that $\mathcal{B}: \mathcal{C} \rightarrow \mathcal{P}(\mathcal{C})$ is completely continuous.

Claim III. $\mathcal{B}$ has a closed graph. Let $x_{n} \rightarrow x_{*}, h_{n} \in \mathcal{B}\left(x_{n}\right)$ and $h_{n} \rightarrow h_{*}$. Then we need to show that $h_{*} \in \mathcal{B}\left(x_{*}\right)$. Associated with $h_{n} \in \mathcal{B}\left(x_{n}\right)$, there exists $f_{n} \in S_{F, x_{n}}$ such that for each $t \in I_{q}$,

$$
\begin{aligned}
h_{n}(t)= & \int_{0}^{t}(t-q s) f_{n}(s) d_{q} s \\
& +\frac{\alpha(1+q) t}{1+q-\alpha\left(\nu^{2}-\mu^{2}\right)} \int_{\mu}^{\nu} \int_{0}^{s}(s-r q) f_{n}(r) d_{q} r d_{q} s \\
& -\frac{(1+q) t}{1+q-\alpha\left(\nu^{2}-\mu^{2}\right)} \int_{0}^{1}(1-q s) f_{n}(s) d_{q} s .
\end{aligned}
$$

Then we have to show that there exists $f_{*} \in S_{F, x_{*}}$ such that for each $t \in I_{q}$,

$$
\begin{aligned}
h_{*}(t)= & \int_{0}^{t}(t-q s) f_{*}(s) d_{q} s \\
& +\frac{\alpha(1+q) t}{1+q-\alpha\left(\nu^{2}-\mu^{2}\right)} \int_{\mu}^{\nu} \int_{0}^{s}(s-r q) f_{*}(r) d_{q} r d_{q} s \\
& -\frac{(1+q) t}{1+q-\alpha\left(\nu^{2}-\mu^{2}\right)} \int_{0}^{1}(1-q s) f_{*}(s) d_{q} s .
\end{aligned}
$$

Let us consider the continuous linear operator $\Theta: L^{1}\left(I_{q}, \mathbb{R}\right) \rightarrow \mathcal{C}$ given by

$$
\begin{aligned}
f \mapsto \Theta(f)(t)= & \int_{0}^{t}(t-q s) f(s) d_{q} s \\
& +\frac{\alpha(1+q) t}{1+q-\alpha\left(\nu^{2}-\mu^{2}\right)} \int_{\mu}^{\nu} \int_{0}^{s}(s-r q) f(r) d_{q} r d_{q} s \\
& -\frac{(1+q) t}{1+q-\alpha\left(\nu^{2}-\mu^{2}\right)} \int_{0}^{1}(1-q s) f(s) d_{q} s .
\end{aligned}
$$

Observe that

$$
\left\|h_{n}(t)-h_{*}(t)\right\|=\| \int_{0}^{t}(t-q s)\left(f_{n}(s)-f_{*}(s)\right) d_{q} s
$$




$$
\begin{aligned}
& +\frac{\alpha(1+q) t}{1+q-\alpha\left(\nu^{2}-\mu^{2}\right)} \int_{\mu}^{\nu} \int_{0}^{s}(s-r q)\left(f_{n}(r)-f_{*}(r)\right) d_{q} r d_{q} s \\
& -\frac{(1+q) t}{1+q-\alpha\left(\nu^{2}-\mu^{2}\right)} \int_{0}^{1}(1-q s)\left(f_{n}(s)-f_{*}(s)\right) d_{q} s \| \rightarrow 0
\end{aligned}
$$

as $n \rightarrow \infty$. Thus, it follows by Lemma 3 that $\Theta \circ S_{F}$ is a closed graph operator. Further, we have $h_{n}(t) \in \Theta\left(S_{F, x_{n}}\right)$. Since $x_{n} \rightarrow x_{*}$, therefore, we have

$$
\begin{aligned}
h_{*}(t)= & \int_{0}^{t}(t-q s) f_{*}(s) d_{q} s \\
& +\frac{\alpha(1+q) t}{1+q-\alpha\left(\nu^{2}-\mu^{2}\right)} \int_{\mu}^{\nu} \int_{0}^{s}(s-r q) f_{*}(r) d_{q} r d_{q} s \\
& -\frac{(1+q) t}{1+q-\alpha\left(\nu^{2}-\mu^{2}\right)} \int_{0}^{1}(1-q s) f_{*}(s) d_{q} s
\end{aligned}
$$

for some $f_{*} \in S_{F, x_{*}}$. Hence $\mathcal{B}$ has a closed graph (and therefore has closed values). In consequence, the operator $\mathcal{B}$ is compact valued.

Thus, the operators $\mathcal{A}$ and $\mathcal{B}$ satisfy hypotheses of Theorem 3 and therefore, by its application, it follows either condition (i) or condition (ii) holds. We show that the conclusion (ii) is not possible. If $x \in \lambda \mathcal{A}(x)+\lambda \mathcal{B}(x)$ for $\lambda \in(0,1)$, then there exists $f \in S_{F, x}$ such that

$$
\begin{aligned}
x(t)= & \lambda \int_{0}^{t}(t-q s) f(s) d_{q} s \\
& +\lambda \frac{\alpha(1+q) t}{1+q-\alpha\left(\nu^{2}-\mu^{2}\right)} \int_{\mu}^{\nu} \int_{0}^{s}(s-r q) f(r) d_{q} r d_{q} s \\
& -\lambda \frac{(1+q) t}{1+q-\alpha\left(\nu^{2}-\mu^{2}\right)} \int_{0}^{1}(1-q s) f(s) d_{q} s \\
& +\lambda\left[1+\frac{(1+q)(\alpha(\nu-\mu)-1) t}{1+q-\alpha\left(\nu^{2}-\mu^{2}\right)}\right]\left(x_{0}+g(x)\right) .
\end{aligned}
$$

Consequently, we have

$$
\begin{aligned}
|x(t)| \leq & \frac{1}{q+1}+\frac{|\alpha|(1+q)}{\left|1+q-\alpha\left(\nu^{2}-\mu^{2}\right)\right|} \int_{\mu}^{\nu} \int_{0}^{s}(s-r q)|f(r)| d_{q} r d_{q} s \\
& +\frac{(1+q)}{\left|1+q-\alpha\left(\nu^{2}-\mu^{2}\right)\right|} \int_{0}^{1}(1-q s)|f(s)| d_{q} s \\
& +\left[1+\frac{(1+q)|\alpha(\nu-\mu)-1|}{\left|1+q-\alpha\left(\nu^{2}-\mu^{2}\right)\right|}\right]\left[\left|x_{0}\right|+\ell\|x\|\right] \\
\leq & \left\{\frac{1}{1+q}+\frac{|\alpha|}{\left|1+q-\alpha\left(\nu^{2}-\mu^{2}\right)\right|} \frac{\left(\nu^{3}-\mu^{3}\right)}{\left(1+q+q^{2}\right)}\right. \\
& \left.+\frac{1}{\left|1+q-\alpha\left(\nu^{2}-\mu^{2}\right)\right|}\right\} \psi(\|x\|)\|p\| \\
& +\left[1+\frac{(1+q)|\alpha(\nu-\mu)-1|}{\left|1+q-\alpha\left(\nu^{2}-\mu^{2}\right)\right|}\right]\left[\left|x_{0}\right|+\ell\|x\|\right] .
\end{aligned}
$$


If condition (ii) of Theorem 1 holds, then there exists $\lambda \in(0,1)$ and $x \in \partial B_{r}$ with $x=\lambda \mathcal{N}(x)$. Then, $x$ is a solution of (4.2) with $\|x\|=M$. Now, by the last inequality, we have

$$
\frac{\left(1-\ell \Lambda_{1}\right) M}{\Lambda_{2} \psi(M)\|p\|+\Lambda_{1}\left|x_{0}\right|} \leq 1
$$

which contradicts (4.1). Hence, $\mathcal{N}$ has a fixed point in $I_{q}$ by Theorem 3 , and consequently the problem (1.2) has a solution. This completes the proof.

Remark 1. Notice that the condition $L_{0}<1$ can be neglected if we use the wellknown Bielecki's renorming method [19]. In this case, the assumption $\left(H_{3}\right)$ can be fixed accordingly.

Remark 2. If $\psi$ satisfies a sublinear condition or more generally

$$
\lim _{\xi \rightarrow \infty} \frac{\xi}{\Lambda_{2} \psi(\xi)\|p\|+\Lambda_{1}\left|x_{0}\right|}>1-\ell \Lambda_{1}
$$

then the existence of $M$ in $\left(H_{4}\right)$ is guaranteed.

Example 3. Consider the following inclusion problem

$$
\left\{\begin{array}{l}
D_{q}^{2} x(t) \in F(t, x(t)), \quad t \in I_{q} \\
x(0)=\frac{1}{3}+\frac{1}{3} x\left(t_{i}\right), \quad x(1)=4 \int_{1 / 3}^{1 / 2} x(s) d_{q} s, \quad t_{i} \in I_{q} .
\end{array}\right.
$$

Here, $x_{0}=1 / 3, \ell=1 / 3, \alpha=4, \mu=1 / 3, \nu=1 / 2$, and $F: I_{q} \times \mathbb{R} \rightarrow \mathcal{P}(\mathbb{R})$ is a multivalued map given by

$$
x \rightarrow F(t, x)=\left[\frac{1}{9} \frac{|x|^{3}}{|x|^{3}+3}, \frac{1}{20} \frac{|x|}{|x|+1}\right] .
$$

For $f \in F$, we have

$$
|f| \leq \max \left(\frac{1}{9} \frac{|x|^{3}}{|x|^{3}+3}, \frac{1}{20} \frac{|x|}{|x|+1}\right) \leq \frac{1}{9}, \quad x \in \mathbb{R} .
$$

Thus,

$$
\|F(t, x)\|_{\mathcal{P}}:=\sup \{|y|: y \in F(t, x)\} \leq \frac{1}{9}=p(t) \psi(\|x\|), \quad x \in \mathbb{R}
$$

with $p(t)=1, \psi(\|x\|)=\frac{1}{9}$. Using the given data together with the condition $\left(H_{4}\right)$, it is found that

$$
M>\frac{19(1+q)+\left(1+q+q^{2}\right)\left(34+75 q+2 q^{2}\right)}{15(1+q)(1+3 q)\left(1+q+q^{2}\right)} .
$$

Clearly, all the conditions of Theorem 4 are satisfied. So there exists at least one solution of the problem (4.5) on $I_{q}$. 


\section{Acknowledgment}

The authors thank the reviewers for their useful and positive comments that led to the improvement of the original manuscript.

\section{References}

[1] C.R. Adams. On the linear ordinary q-difference equation. Ann. of Math., 30:195-205, 1928. http://dx.doi.org/10.2307/1968274.

[2] B. Ahmad. Boundary value problems for nonlinear third-order $q$-difference equations. Electron. J. Differential Equations, 94:1-7, 2011.

[3] B. Ahmad, A. Alsaedi and S.K. Ntouyas. A study of second-order $q$-difference equations with boundary conditions. Adv. Difference Equ., 35:1-10, 2012.

[4] B. Ahmad and J.J. Nieto. Basic theory of nonlinear third-order $q$-difference equations and inclusions. Math. Model. Anal., 18:122-135, 2013. http://dx.doi.org/10.3846/13926292.2013.760012.

[5] B. Ahmad and S.K. Ntouyas. Boundary value problems for $q$-difference inclusions. Abstr. Appl. Anal., 2011:1-15, 2011.

[6] M.H. Annaby and Z.S. Mansour. q-taylor and interpolation series for jackson q-difference operators. J. Math. Anal. Appl., 344:472-483, 2008. http://dx.doi.org/10.1016/j.jmaa.2008.02.033.

[7] M.H. Annaby and Z.S. Mansour. q-Fractional Calculus and Equations, volume 2056 of Lecture Notes in Mathematics. Springer-Verlag, Berlin, 2012.

[8] G. Bangerezako. Variational q-calculus. J. Math. Anal. Appl., 289:650-665, 2004. http://dx.doi.org/10.1016/j.jmaa.2003.09.004.

[9] L. Byszewski. Theorems about existence and uniqueness of solutions of a semilinear evolution nonlocal Cauchy problem. J. Math. Anal. Appl., 162:494-505, 1991. http://dx.doi.org/10.1016/0022-247X(91)90164-U.

[10] L. Byszewski and V. Lakshmikantham. Theorem about the existence and uniqueness of a solution of a nonlocal abstract Cauchy problem in a Banach space. Appl. Anal., 40:11-19, 1991. http://dx.doi.org/10.1080/00036819008839989.

[11] R.D. Carmichael. The general theory of linear $q$-difference equations. Amer. J. Math., 34:147-168, 1912. http://dx.doi.org/10.2307/2369887.

[12] K. Deimling. Multivalued Differential Equations. Walter De Gruyter, Berlin, New York, 1992.

[13] A. Dobrogowska and A. Odzijewicz. Second order $q$-difference equations solvable by factorization method. J. Comput. Appl. Math., 193:319-346, 2006. http://dx.doi.org/10.1016/j.cam.2005.06.009.

[14] M. El-Shahed and H. A. Hassan. Positive solutions of $q$-difference equation. Proc. Amer. Math. Soc., 138:1733-1738, 2010. http://dx.doi.org/10.1090/S0002-9939-09-10185-5.

[15] T. Ernst. The history of q-calculus and a new method. UUDMReport2000:16. Department of Mathematics, Uppsala University, 2000.

[16] R. Ferreira. Nontrivial solutions for fractional $q$-difference boundary value problems. Electron. J. Qual. Theory Differ. Equ., 70:1-10, 2010. 
[17] G. Gasper and M. Rahman. Basic Hypergeometric Series. Cambridge University Press, Cambridge, 1990.

[18] G. Gasper and M. Rahman. Some systems of multivariable orthogonal $q$-Racah polynomials. Ramanujan J., 13:389-405, 2007.

http://dx.doi.org/10.1007/s11139-006-0259-8.

[19] A. Granas and J. Dugundji. Fixed Point Theory. Springer-Verlag, New York, 2005.

[20] Sh. Hu and N. Papageorgiou. Handbook of Multivalued Analysis, Theory I. Kluwer, Dordrecht, 1997.

[21] M.E.H. Ismail and P. Simeonov. $q$-difference operators for orthogonal polynomials. J. Comput. Appl. Math., 233:749-761, 2009. http://dx.doi.org/10.1016/j.cam.2009.02.044.

[22] F.H. Jackson. On $q$-functions and a certain difference operator,. Trans. Roy. Soc. Edinburgh, 46:253-281, 1908. http://dx.doi.org/10.1017/S0080456800002751.

[23] F.H. Jackson. On q-difference equations. Amer. J. Math., 32:305-314, 1910. http://dx.doi.org/10.2307/2370183.

[24] V. Kac and P. Cheung. Quantum Calculus. Springer, New York, 2002.

[25] A. Lasota and Z. Opial. An application of the kakutani-ky fan theorem in the theory of ordinary differential equations. Bull. Acad. Polon. Sci. Ser. Sci. Math. Astronom. Phys., 13:781-786, 1965.

[26] J. Ma and J. Yang. Existence of solutions for multi-point boundary value problem of fractional q-difference equation. Electron. J. Qual. Theory Differ. Equ., 92:1$10,2011$.

[27] T.E. Mason. On properties of the solutions of linear $q$-difference equations with entire function coefficients. Amer. J. Math., 37:439-444, 1915.

http://dx.doi.org/10.2307/2370216.

[28] D. O'Regan. Fixed-point theory for the sum of two operators. Appl. Math. Lett., 9:1-8, 1996. http://dx.doi.org/10.1016/0893-9659(95)00093-3.

[29] W.V. Petryshyn and P.M. Fitzpatric. A degree theory, fixed point theorems, and mapping theorems for multivalued noncompact maps. Trans. Amer. Math. Soc., 194:1-25, 1974. http://dx.doi.org/10.1090/S0002-9947-1974-2478129-5. 